\title{
DE AGNA HAEDOQUE FAUNO IMMOLANDIS (HOR. CARM. 1, 4, 11-12)
}

\section{Elias R. Gimadeev}

Moscow State University, Leninskie gory, 1, str. 51, Moscow, 119234, Russian Federation; eliasgimad@mail.ru

The author focuses his analysis on the sacrifice of an ewe lamb and/or a buckling mentioned in the problematic locus of Horace. He notices that the expression agna sive haedo occurs only twice in Latin literature. Both times it is used by Horace (which probably explains the dark Servius auctus' comment to the similar usage by Virgil, Aen. 8, 641). Basing on different ancient sources and secondary literature, the author comes to conclusion that the usage of agna with haedus in the sacrifice in honor of Faunus, notwithstanding the Roman custom, which prescribed male victims for male gods, relates to the poetic language of Horace. This explanation virtually differs from that proposed by R. G. M. Nisbet and M. Hubbard, who tend to explain this double sacrifice by the Greek tradition of sacrificial offering to Priapus. Supporting his conclusion the author also reveals that in certain cases Romans did sacrifice female victims to male gods, especially in the official cult (hostia honoraria). Refs 7.

Keywords: Horace, odes, sacrifice in ancient Rom, sacrificial animals.

Inter nonnulla sacrificiorum specimina Horatiana immolationis exemplum eximium in Carm. 1, 4, 11-12 invenitur. Dicit poeta ritum ineunte vere agnam aut haedum Fauno sacrificandi exstitisse:

$$
\begin{aligned}
& \text { nunc [vere scil.] et in umbrosis Fauno decet immolare lucis, } \\
& \text { seu poscat agna sive malit haedo. }
\end{aligned}
$$

Vix veri simile fortasse videatur vates Venusinus nullo modo de duorum animalium sacrificio pro alio quodam ritu temere i. e. licentia poetica nixus vel metri causa commemorasse. Imprimis ad commentarium Pomponi Porphyrionis referendum est, qui bis apud Horatium victimas feminini generis pro masculino et semel mutato sexu adnotavit, ${ }^{1}$ quod minime moris Romanorum esse videtur. Ad vv. 11-12 talia enarrat grammaticus: Adtende feminino genere agnam maluisse dicere quam agnum, secundum illud Vergilianum: Et caesa iungebant foedera porca $($ Aen. 8,641$) .^{2}$ Nescio quid enim quaedam eloquutiones per femininum genus gratiores fiunt. Cum autem Porphyrio nec non Pseudacro, in cuius libro de moribus solum dis agrestibus ineunte anno sacrificandi tractatur (ad v. 11), ${ }^{3}$ de hostiis ipsis taceant, sententiam admodum audaciorem de Servii aucti scholiis (SD ad Aen. 8, 641) afferamus: falso autem ait 'porca': nam ad hoc genus sacrificii porcus adhibebatur. ergo

${ }^{1}$ Cf. Porfyr. ad Epod. 9, 22: et intactas boves, id est: indomitas. Feminino autem genere boues non sic dixit, quasi feminis tantum triumphantes sacrificent, sed quia nescio quid gratius feminino genere boues quam masculino dicuntur. Denique et Vergilius: Ille meas errare boues, ut cernis (Ecl. 1, 9); ad Epist. 2, 1, 143: tellvrem porco, pro porca. Vt e contrario Verg(ilius): Et caesa iungebant foedera porca (Aen. 8, 641).

${ }^{2}$ Verg. Aen. 8, 641: h.l. reges ante Iovis aram sacrificium faciunt.

${ }^{3}$ Quasi expleto anno et novo redeunte reddenda sunt diis agrestibus vota, ut Vergilius (Georg. 1, 341): Tum pingues agni et tum mollissima vina.

(C) St. Petersburg State University, 2017 
aut usurpavit genus pro genere, ut timidi 'venient ad pocula dammae', cum has dammas dicamus, item supra 'lupam', cum artis sit 'hic' et 'haec lupus': aut certe illud ostendit, quia in omnibus sacris feminini generis plus valent victimae. denique si per marem litare non possent, succidanea dabatur femina; si autem per feminam non litassent, succidanea adhiberi non poterat. quidam 'porcam' euphoniae gratia dictam volunt.

Itaque ex certissimo fontium testimonio constat atque Arnobii auctoritate affirmatur $($ Nat. 7, 19) Romanos dis maribus masculini sexus pecus mactasse, deas plerumque feminini sexus animalibus coluisse, qua de causa hostiae sexus scrupulum Deuteroservio iniecit, ${ }^{4}$ quod 'porcam' euphoniae gratia dictam intellexit. In his, quae a viro doctissimo dicta sunt, etiam Aulus Gellius $(13,21,14)^{5}$ et ipse quidem Quintilianus $(8,3,19)^{6}$ assentiuntur. Hic enim Vergilium, cum nomen porcae pro porco dixerit, non rationem magis, quam sensum sequi, quia 'porca' bene sonet, arbitratur, ille autem in commentario suo ad Ennium cum Quintiliano de feminini generis usu consentit atque affirmat Ennium verbis aere fulva pro aere fulvo uti non solum ad Graecorum modum dicendi imitandum (cf. ap.

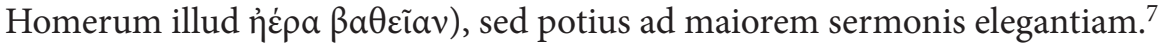

Itaque cum scholiastae de re nostra nihil fere tradant, ad commentatores recentioris aetatis nos conferamus. Orellius, lumen philologiae, verbum immolare et cum accusativo et cum ablativo construi posse explanat interque agnam haedumve in sacrificando discrimen nullius momenti esse putat. Idem etiam Kiesslingius et Heinzius. Quibus viris doctissimis maxime Nisbetius et Hubbardia dissentiunt, quibus primam carminis partem, ubi de veris adventu agitur, ex Alcaeo ( $f r .286)$ vel ex Leonida Tarentino ( $A P$ 10, 1) Horatius deprompsisse videtur, cum Leonidas aliorumque Graecorum nonnulli de Priapo fertilitatis fautore navigationumque patrono narrent $(A P 10,2 ; 4 ; 5 ; 6 ; 14 ; 15$;

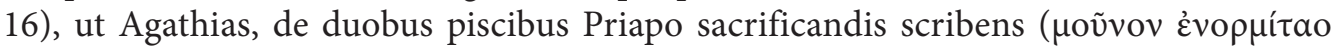

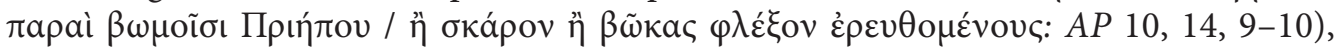

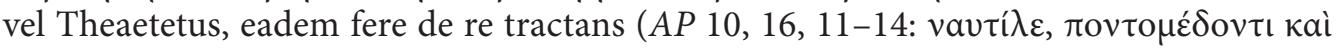

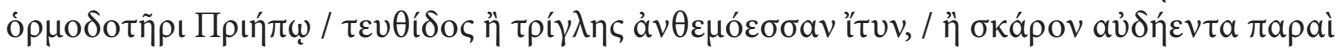

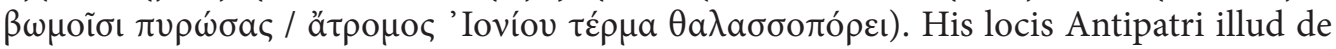

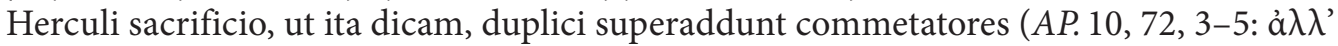

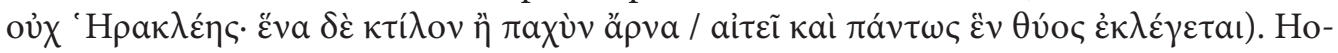
rum locorum et similium auctoritate innitentes putant Nisbetius Hubbardiaque Priapum Graecum adaequatum esse apud Horatium Italico Fauno, fertilitate augenda videlicet, non tamen nautarum securitate functum deum. ${ }^{8}$ Totum itaque immolationis ordinem secundum Graecos descriptum esse apud Venusinum poetam ritu Romanorum neglecto. Hanc sententiam prorsus negare non possum, litem autem sub iudice adhuc esse puto.

${ }^{4}$ Arnob. Nat. 7, 252: Sed erras, inquit, et laberis: nam dis feminis feminas, mares maribus hostias immolare abstrusa et interior ratio est vulgique a cognitione dimota; ibid. 253: sexibus sexus pares, id est feminas feminis, mares autem hostias dis maribus immolari sacrificiorum iura praescribunt. Cf. ThLL 1, 1363, 45 sqq. et 1365,69 sqq.

${ }_{5}$ Mentionem ap. Mastellone 1994, 127 inveni. Gell. 13, 21, 14: Contra vero idem Ennius in annali du-

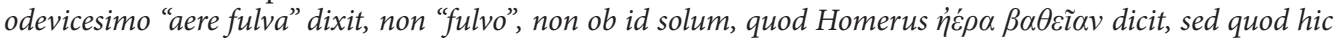
sonus, opinor, vocabilior est visus et amoenior.

${ }^{6}$ Quint. 8, 3, 19: Quaedam non tam ratione quam sensu iudicantur, ut illud "caesa iungebant foedera porca" fecit elegans fictio nominis, quod si fuisset "porco" vile erat.

7 De aliis causis generis deligendi invenies ap. Diederich, 1999, 183-184 et Mastellone 1994, 01-128.

${ }^{8}$ Nisbet, Hubbard 1970, 60: "It looks as if in Horace's ode Faunus, though he has no connection with navigation, in some way represents Priapus; both gods were connected with the fertility of the animal kingdom". 
Ad fidem huic opinioni conciliandam conducit etiam Crausii dissertatio. Ille enim ritu Graeco deabus feminina animalia, dis tamen hostiae utriusque generis mactata esse existimat. ${ }^{9}$ Attamen testimonia poetarum, qui sane haud raro feminas dis, mares deabus mactari referant, pro suspectis habet. ${ }^{10}$ Illos enim testes partim arcana pontificalis disciplinae arte satis imbutos non fuisse, ut non in rebus remotis errarent, partim sacrificia privata referre existimat. ${ }^{11}$ Adnotavit etiam v. d. canem aut caprum Fauno Lupercalibus a Lupercis (Plut. QR 68. 111; Rom. 21. Valer. Max. 2, 2, 9), in privatis autem sacrificiis haedum et ovem, feminini scilicet sexus, et agnam (Hor. Carm. 2, 18, 5; 17, 32; Ovid. Fast. 4, 653) sacrificari posse. ${ }^{12}$

Omnes fere commentatores hanc Fauno immolationem secundum Ovidianum Idibus agrestis fumant altaria Fauni (Fast. 2, 193, cf. CIL VI 2302) Idibus Februariis conjunxerunt. Nisbetius Hubbardiaque hanc sententiam item probaverunt, cum Idibus Februariis honore Fauno debito tributo ad vesperum dies parentales incepissent, qui viris doctis cum morte pallida apud Horatium v. 13 conjuncti esse videntur. ${ }^{13}$ Idibus Februariis dies natalis templi Fauno consecrati Romae in insula Tiberina (Fasti Esquil., inscr. Ital. 13, 2, 32 et vide Ov. l. l.) celebrabatur. Mihi autem permirum videtur poeta ruri ad fontem Albuneae in agro Tiburtino eundem diem festum celebrasse, quem et Romae. Nisbetius Hubbardiaque quaestionem posuerunt, sed certum responsum non dant. Inscriptiones nulla testimonia certa de sacrificiis privatis Fauno procurandis servaverunt. ${ }^{14}$ Huc addendum est Fauno a. d. XV Kal. Mart. Lupercalibus, quae dies ad Faunum in ultima antiquitate non pertinebant, ${ }^{15}$ a Lupercis canem caprumve, ut supra dixi, mactatos esse. Fortasse non solum Idibus Februariis, sed etiam a. d. XV Kal. Mart., Lupercalibus scilicet, agnam aut haedum privatim sine ullo graviore praescripto mactari potuisse.

Itaque ad conclusiones nos referamus.

1. Nemo commentatorum mentionem fecisse verbum agnae una cum verbo haedi a Horatio iam in Epod. 2, $59^{16}$ usurpatum esse: vel agna festis caesa Terminalibus / vel haedus ereptus lupo. Cum nusquam auctores antiqui his duobus verbis una utantur, credo affirmoque Horatium loco tractato sui ipsius verbis olim feliciter inventis denuo usum esse non scrupulosae rituum descriptionis causa, ${ }^{17}$ sed sermonis elegantiae cuiusdam gratia, nobis vero subobscurae, quod nobis Serviana euphonia in genere feminino utendo explanat.

2. Duplicitas Fauno datae hostiae explicata esse potest contra Nisbetium Hubbardiamque ex more Romanorum publice una cum sacrificiis sollemnibus piacularia et honoraria facere. ${ }^{18}$ Antiquissima aetate hostias tenuiores mactare solebant, tempore posteriore sac-

${ }^{9}$ Krause $1894,19$.

${ }^{10}$ Cf.: Latte 1960, 380: "Der Versuch von K. Krause, die entgegenstehenden Fälle zu beseitigen, ist gewaltsam".

11 Ibid. 22-23.

12 Ibid. 32.

13 Nisbet, Hubbard 1970, 66 sq.

14 Wissowa 1912, 175. Silvanus contra more Romano in cultu privato colebatur, cui testimonia permulta habemus. Wissowa, 1912, 176. Hor. Epist. 2, 1, 143 et Juven. 6, 447 de hoc genere sacrificiorum Silvano procurandorum cantant.

${ }^{15}$ Latte $1960,87$.

16 Mankin 1995, 84 commentarium Nisbetii Hubbardiaeque ad Hor. Carm. 1, 4, 12 conferre jubet.

17 Cf.: Ovid. Fast. 2, 361 sq.: cornipedi Fauno caesa de more capella / venit ad exiguas turba vocata dapes; Plut. Rom. 21. Praeter hoc sacrificium duplex agno et porca deo Termino ap. Ovid. Fast. 2, 655 sq.

18 Wissowa 1912, 349, 3. 
rificiis sollemnibus, ne iniuriam dis deabusque inferrent, honoraria nonnulla addiderunt. Fratres Arvales quidem non solum agnam optimam ac porcilias piaculares, sed etiam vaccam honorariam albam caedebant. Non aliter Iovi et Iunoni non solum bos, sed etiam ovis sive agna mactabatur. ${ }^{19}$ Plurimum interest ad religiones Romanorum cognoscendas duplex sacrificium dis deabusque masculini ac feminini generis animalibus omnino fieri potuisse. Similia in cultu privato extitisse, quamvis poeta nullum huius generis ritum $l$. $c$. describat, arbitramur. Quae cum ita sint, Horatiana haec sermonis elegantia lectori eius vel minime inusitata videbatur.

\section{Opera citata}

Diederich S. Der Horazkommentar des Porphyrio im Rahmen der kaiserzeitlichen Schul-und Bildungstradition. Berlin - New York, De Gruyter, 1999.

Krause C. De Romanorum hostiis quaestiones selectae. Diss. Marpurgi Cattorum, 1894.

Latte K. Römische Religionsgeschichte. München, C.H. Beck, 1960.

Mankin D. (ed.) Horace. Epodes. Cambridge, CUP, 1995.

Mastellone I. E. Osservazioni di lingua nel commento di Porfirione ad Orazio. Bolletino di Studi Latini 1994, 24, 101-128.

Nisbet R. G. M., Hubbard M. (eds) A Commentary on Horace. Odes. Book I. Oxford, Clarendon Press, 1970. Wissowa G. Religion und Kultus der Römer. München, C. H. Beck, ${ }^{2} 1912$.

For citation: Elias R. Gimadeev. De agna haedoque Fauno immolandis (Hor. Carm. 1, 4, 11-12). Philologia Classica 2017, 12(1), 115-118. DOI: 10.21638/11701/spbu20.2017.112.

\section{О ЖЕРТВОПРИНОШЕНИИ ФАВНУ ОВЕЧКОЙ И КОЗЛЕНКОМ (HOR. CARM. 1, 4, 11-12).}

Ильяс Рустэмович Гимадеев

Московский Государственный Университет, Российская Федерация, 119234, Москва, Ленинские горы 1, стр. 51; eliasgimad@mail.ru

В статье рассматривается проблема жертвоприношения в вышеназванных стихах Горация. Автор обнаруживает, что выражение agna sive haedo употребляется дважды и только у Горация (что, вероятно, объясняет комментарий Девтеро-Сервия к похожему месту у Вергилия, Aen. 8, 641). Исследовав материал источников и литературу вопроса, автор заключает, что употребление agna вместе c haedus характерно для Горация. Вывод статьи кардинально расходится с мнением Р. Нисбета и М.Хаббард, которые объясняют данное двойное жертвоприношение у Горация греческим обычаем приносить двойную жертву Приапу. Тезис автора подтверждается тем обстоятельством, что в государственном культе римляне могли изредка приносить в жертву божеству мужского пола животное женского пола, особенно в качестве hostia honoraria. Библиогр. 7 назв.

Ключевые слова: Гораций, оды, жертвоприношение в Древнем Риме, жертвенные животные. 